\title{
Worldwide Effects of Coronavirus Disease Pandemic on Tuberculosis Services, January-April 2020
}

Giovanni Battista Migliori, ${ }^{1}$ Pei Min Thong, ${ }^{1}$ Onno Akkerman, Jan-Willem Alffenaar, Fernando Álvarez-Navascués, Mourtala Mohamed Assao-Neino, Pascale Valérie Bernard, Joshua Sorba Biala, François-Xavier Blanc, Elena M. Bogorodskaya, Sergey Borisov, Danilo Buonsenso, Marianne Calnan, Paola Francesca Castellotti, Rosella Centis, Jeremiah Muhwa Chakaya, Jin-Gun Cho, Luigi Ruffo Codecasa, Lia D’Ambrosio, Justin Denholm, Martin Enwerem, Maurizio Ferrarese,

Tatiana Galvão, Marta García-Clemente, José-María García-García, Gina Gualano, José Antonio Gullón-Blanco, Sandra Inwentarz, Giuseppe Ippolito, Heinke Kunst, Andrei Maryandyshev, Mario Melazzini,

Fernanda Carvalho de Queiroz Mello, Marcela Muñoz-Torrico, Patrick Bung Njungfiyini, Domingo Juan Palmero, Fabrizio Palmieri, Pavilio Piccioni, Alberto Piubello, Adrian Rendon, Josefina Sabriá, Matteo Saporiti, Paola Scognamiglio, Samridhi Sharma, Denise Rossato Silva, Mahamadou Bassirou Souleymane, Antonio Spanevello, Eva Tabernero, Marina Tadolini, Michel Eke Tchangou, Alice Boi Yatta Thornton, Simon Tiberi, Zarir F. Udwadia, Giovanni Sotgiu, Catherine Wei Min Ong, ${ }^{2}$ Delia Goletti ${ }^{2}$

Author affiliations: Istituti Clinici Scientifici Maugeri IRCCS, Tradate, Italy (G.B. Migliori, R. Centis, A. Spanevello); Yong Loo Lin School of Medicine, National University of Singapore, Singapore (P.M. Thong, C.W.M. Ong); University of Groningen, Groningen, the Netherlands (O. Akkerman); The University of Sydney, Sydney, New South Wales, Australia (J.-W. Alffenaar, J.-G. Cho); Westmead Hospital, Sydney (J.-W. Alffenaar, J.-G. Cho); Hospital Universitario San Agustín, Avilés, Spain (F. Álvarez-Navascués, J.A. Gullón-Blanco); National Tuberculosis Programme, Niamey, Niger (M.M. Assao-Neino); Centre Hospitalier Universitaire, Nantes, France (P.V. Bernard, F.-X. Blanc); Community Health Centre, Tombo, Sierra Leone (J.S. Biala); Moscow Research and Clinical Center for TB Control, Moscow, Russia (E.M. Bogorodskaya, S. Borisov); Fondazione Policlinico Universitario A. Gemelli IRCCS, Rome, Italy (D. Buonsenso); University Research Co. LLC, Manila, the Philippines (M. Calnan); Niguarda Hospital, Milan, Italy (P.F. Castellotti, L.R. Codecasa, M. Ferrarese, M. Saporiti); Kenyatta University, Nairobi, Kenya (J.M. Chakaya); Liverpool School of Tropical Medicine, Liverpool, UK (J.M. Chakaya); Parramatta Chest Clinic, Parramatta, New South Wales, Australia (J.-G. Cho); Public Health Consulting Group, Lugano, Switzerland (L. D’Ambrosio); Melbourne Health Victorian Tuberculosis Program, Melbourne, Victoria, Australia (J. Denholm); University of Melbourne, Melbourne (J. Denholm); Amity Health Consortium, Johannesburg, South Africa (M. Enwerem); Universidade Federal da Bahia, Salvador, Brazil (T. Galvão); Hospital Universitario Central de Asturias, Oviedo, Spain (M. GarcíaClemente); Tuberculosis Research Programme SEPAR, Barcelona, Spain (J.-M. García-García); National Institute for Infectious Diseases 'L. Spallanzani' IRCCS, Rome (G. Gualano, G. Ippolito, F. Palmieri,
P. Scognamiglio, D. Goletti); Instituto Vaccarezza, Buenos Aires, Argentina (S. Inwentarz, D.J. Palmero); Royal London Hospital of Barts Health National Health Service Trust, London, UK (H. Kunst, S. Tiberi); Queen Mary University, London (H. Kunst, S. Tiberi); Northern State Medical University, Arkhangelsk, Russian Federation (A. Maryandyshev); Istituti Clinici Scientifici Maugeri IRCCS, Pavia, Italy (M. Melazzini); Instituto de Doenças do Tórax, Universidade Federal do Rio de Janeiro, Rio de Janeiro, Brazil (F.C.Q. Mello); Instituto Nacional De Enfermedades Respiratorias Ismael Cosio Villegas, Mexico City, Mexico (M. Muñoz-Torrico); Community Health Center, Hastings, Sierra Leone (P.B. Njungfiyini; M.E. Tchangou); Amedeo di Savoia Hospital, Turin, Italy (P. Piccioni); Damien Foundation, Niamey, Niger (A. Piubello, M.B. Souleymane); University Hospital of Monterrey (Universidad Autonoma de Nuevo Leon), Monterrey, Mexico (A. Rendon); Hospital Transversal Moises Broggi-HGH, Barcelona (J. Sabriá); P.D. Hinduja National Hospital and Medical Research Centre, Mumbai, India (S. Sharma, Z.F. Udwadia); Universidade Federal do Rio Grande do Sul (UFRGS), Porto Alegre, Brazil (D.R. Silva); University of Insubria, Varese-Como, Italy (A. Spanevello); Hospital de Cruces, Vizcaya, Spain (E. Tabernero); Unit of Infectious Diseases Alma Mater Studiorum University of Bologna, Bologna, Italy (M. Tadolini); Saint John of God Catholic Hospital, Mabesseneh Lunsar, Sierra Leone (A.B.Y. Thornton); University of Sassari, Sassari, Italy (G. Sotgiu); National University of Singapore, Singapore (C.W.M. Ong)

DOI: https://doi.org/10.3201/eid2611.203163

${ }^{1}$ These first authors contributed equally to this article.

${ }^{2}$ These senior authors contributed equally to this article. 
Coronavirus disease has disrupted tuberculosis services globally. Data from 33 centers in 16 countries on 5 continents showed that attendance at tuberculosis centers was lower during the first 4 months of the pandemic in 2020 than for the same period in 2019. Resources are needed to ensure tuberculosis care continuity during the pandemic.

$\mathrm{T}$ he coronavirus disease (COVID-19) pandemic has affected clinical management of tuberculosis (TB) and TB-related services $(1,2)$. Reports of the first cohorts of patients with COVID-19 and TB have been recently published $(3,4)$, although it may be difficult to distinguish which infection occurred first (5). The effects of COVID-19 on TB diagnostic and programmatic activities are similar (1). Almost every country has national TB programs in place, whereas national programs for COVID-19 are urgently needed $(1,2)$.

The effect of COVID-19 on TB services is estimated to be dramatic, especially in countries where healthcare staff involved in TB management have been reassigned to the COVID-19 emergency. However, apart from local studies (6), a comprehensive, multinational description is needed.

The Global Tuberculosis Network, which conducted this study, collaborates with TB centers from 41 countries $(3,4,6,7)$. We studied patient attendance at TB centers in 16 countries and compared the volume of TB-related healthcare activities in the first 4 months of the COVID-19 pandemic, January-April 2020, with that for the same period in 2019.

\section{The Study}

We invited 37 TB centers to participate in the study and collected data from 33 centers located in 16 countries on 5 continents (Appendix Tables 1, 2, https:/ / wwwnc.cdc.gov/EID/article/26/11/20-3163-App1. pdf). The participating centers received ethics clearance according to their respective center regulations $(7,8)$. Active TB disease and latent TB infection (LTBI) were defined according to international guidelines $(9,10)$. We recorded numbers of patients with active TB discharged from inpatient care, patients with newly diagnosed cases of active TB, patients with active TB visiting outpatient settings, and new and total outpatient visits for LTBI. We defined use of telehealth services as implementation of directly observed therapy during face-to-face virtual teleconsultations, which were considered to be equivalent to outpatient visits and were counted as such. We did not consider patient contact by telephone and emails to be telehealth. Home visits were considered outpatient visits. We also recorded national lockdown dates. If a country reported results from $>1$ center, we used the sum of the attendances to generate the graphs. Quantitative variables were summarized with absolute (percentage) frequencies.

Of the 16 countries studied, data were contributed by 4 TB centers each in Italy, Russia, Spain, and Brazil; 3 each in Sierra Leone and Niger; 2 in Mexico; and 1 each in 9 other countries (Appendix Tables 1, 2). Lockdowns were imposed in all countries (Appendix Figures 1, 2). The earliest lockdown start date was February 1, 2020 (Australia); the latest was April 7, 2020 (Singapore). By the end of data collection (April 30), none of the 16 countries had reduced lockdown severity.

Data on new active TB cases were available from 32 of the 33 TB centers. Except for 5 centers (Sydney, New South Wales, Australia; San Fernando, the Philippines; Turin, Italy; Asturias, Spain; and London, UK), which each reported stable numbers or moderate increases, new active TB cases decreased in $27(84 \%)$ of the 32 TB centers in the first 4 months of 2020 relative to the same period in 2019 (Appendix Figure 1).

Information about total outpatient $\mathrm{TB}$ visits was available for 29 centers but not from Groningen, the Netherlands; Mexico City, Mexico; Porto Alegre, Brazil, and Nairobi, Kenya. A total of 22 (75\%) of 29 TB centers from 14 countries registered decreased outpatient visits during the lockdowns.

Active TB-associated hospital discharges differed in 2020 from 2019. Although data were not available for a few centers (Buenos Aires, Argentina; Nairobi; and the 3 centers in Niger), data for San Fernando, Singapore; Mexico City, Groningen, and London indicated minimal or no increase. Active TB-associated hospital discharges for the remaining 23 (82\%) of $28 \mathrm{~TB}$ centers were lower during the first 4 months of 2020 .

Data for LTBI outpatient visits were available from 16 of the 33 TB centers; 13 (81\%) recorded decreased total outpatient visits (all except Hastings, Sierra Leone; Alvorada, Brazil; and Barcelona, Spain) (Appendix Figure 2). Data for newly diagnosed LTBIs were available from 19 of the 33 TB centers. New LTBI outpatient visits at 18 (95\%) of 19 TB centers (all except Alvorada) were fewer during the lockdown period (Appendix Figure 2).

During the first 4 months of 2020, telehealth services were used by $7(21 \%)$ of the 33 TB centers. The number of patients using telehealth services was reported by 4 centers: Sydney; Mumbai, India; London; and Arkhangelsk, Russia. Increased use of telehealth services correlated with lockdown implementation; most uses were recorded in April 2020 (Appendix Tables 1, 2). 


\section{Conclusions}

For most TB centers during their respective national lockdowns in the first 4 months of 2020, we found reductions in TB-related hospital discharges, newly diagnosed cases of active TB, total active TB outpatient visits, and new LTBI and LTBI outpatient visits. These results may be explained by a general decrease in the use of health services, including emergency services (11). Resources for TB service provision were reassigned to other medical services. Outpatient visit numbers may have decreased because of patients' fear of exposure to severe acute respiratory syndrome coronavirus 2 (12). Access to medical services may have decreased because of interruptions in or difficulty accessing public transportation, although health-related travel was permitted in most countries. In some TB centers (e.g., Mexico City), the hospital patient intake system was modified to support COVID-19 admissions, thus severely hindering TB services. In some centers, screening for LTBI was considered a lower priority than screening for active TB or COVID-19. Because of lockdowns, reactivation of active TB in persons with LTBI who did not receive preventive therapy may be expected, such as in contacts recently exposed to TB or in those who are immunocompromised $(13,14)$. In England, compared with 2019, TB notifications decreased by $16.5 \%$ during April and by $37.3 \%$ during May 2020; the LTBI program was paused in response to COVID-19 on March 26 (15).

Lockdowns have favored the increased use of telemedicine. Telehealth is a new service offered by TB programs. In TB centers surveyed in Australia, Russia, India, and the United Kingdom, telehealth service use increased in the first 4 months of 2020.

Although our study cannot comprehensively describe all features of TB management, we found that the COVID-19 pandemic had a substantial impact on TB services worldwide. The main strength of our study is the global coverage from 33 TB centers from 16 countries on 5 continents. Limitations include lack of data from some countries. In 9 of the 16 countries, data were limited to reports from only 1 TB center, which may not have fully represented that nation's TB healthcare activities. In addition, some TB centers were located in countries with low TB incidence (e.g., Italy). The description of the changes in the TB burden over a few months did not allow for appropriate statistical inferences in these countries with low TB incidence. More information about the medium- and long-term effects of the COVID-19 pandemic on TB services after a specified time from the diagnosis of the first COVID-19 patient in each country is needed.

The COVID-19 pandemic seems to have affected TB services in all 16 countries that provided data.
At select TB centers, increased use of telehealth services during the pandemic was recorded. Resources urgently need to be channeled to ensure that TB care continues efficiently despite the ongoing COVID-19 pandemic.

\section{Acknowledgments}

We express our gratitude to Tauhidul Islam, Thomas Hiatt, and Rajendra Yadav for their critical input and to Pascale Bémer, Julie Coutherut, Emmanuel Eschapasse, Aurélie Guillouzouic, Carole Hervé, Maeva Lefebvre, Chan Ngohou, and Roberta Marques Aguiar for supporting the data.

D.G. is a professor at UNICAMILLUS International University of Health and Medical Sciences in Rome. C.W.M.O. is funded by the Singapore National Medical Research Council (NMRC/TA/0042/2015, CSAINV17nov014) iHealthtech in National University of Singapore, and the National University Health System (NUHS/RO/2017/092/SU/01, CFGFY18P11, NUHSRO/2020/042/RO5+5/ad-hoc/1) in Singapore and is a recipient of the Young Investigator Award, Institut Mérieux, Lyon, France. D.B. received a grant from Cassa Galeno 2019 to develop a research network in Sierra Leone.

The article is part of the activities of the Global Tuberculosis Network of the European Tuberculosis Research Initiative, supported by the World Health Organization Regional Office for Europe and the World Health Organization Collaborating Centre for Tuberculosis and Lung Diseases, Tradate, Italy (ITA-80, 2017-2020-GBM/RC/LDA). Part of the work was supported by Ricerca Corrente (Linea 1 and Linea 4; GR-2018-12367178, GR-2016-02364014) from Italia Ministry of Health.

\section{About the Author}

Prof. Migliori is director of the World Health Organization Collaborating Centre for Tuberculosis and Lung diseases at Istituti Clinici Scientifici Maugeri, Tradate, Italy, and chair of the Global Tuberculosis Network. Primary research interests are prevention, diagnosis, treatment, and rehabilitation of $\mathrm{TB}$, including its relationship with COVID-19.

\section{References}

1. Dara M, Sotgiu G, Reichler MR, Chiang CY, Chee CBE, Migliori GB. New diseases and old threats: lessons from tuberculosis for the COVID-19 response. Int J Tuberc Lung Dis. 2020;24:544-5. https:/ / doi.org/10.5588/ijtld.20.0151

2. Alagna R, Besozzi G, Codecasa LR, Gori A, Migliori GB, Raviglione M, et al. Celebrating World Tuberculosis Day at the time of COVID-19. Eur Respir J. 2020;55:2000650. https:/ / doi.org/10.1183/13993003.00650-2020

3. Motta I, Centis R, D'Ambrosio L, García-García JM, Goletti D, Gualano G, et al. Tuberculosis, COVID-19 and migrants: preliminary analysis of deaths occurring in 69 
patients from two cohorts. Pulmonology. 2020;26:233-40. https://doi.org/10.1016/j.pulmoe.2020.05.002

4. Tadolini M, Codecasa LR, García-García JM, Blanc FX, Borisov S, Alffenaar JW, et al. Active tuberculosis, sequelae and COVID-19 co-infection: first cohort of 49 cases. Eur Respir J. 2020;56:2001398. https:/ / doi.org/10.1183/13993003. 01398-2020

5. Tadolini M, Garcia-Garcia JM, Blanc FX, Borisov S, Goletti D, Motta I, et al. On tuberculosis and COVID-19 co-infection [cited 2020 Aug 13]. https:/ / erj.ersjournals.com/ content/early/2020/06/18/13993003.02328-2020

6. Buonsenso D, Iodice F, Sorba Biala J, Goletti D. COVID-19 effects on tuberculosis care in Sierra Leone. Pulmonology [cited 2020 Aug 13]. https:/ / www.journalpulmonology. org/en-covid-19-effects-on-tuberculosis-care-avanceS2531043720301306

7. Borisov S, Danila E, Maryandyshev A, Dalcolmo M, Miliauskas S, Kuksa L, et al. Surveillance of adverse events in the treatment of drug-resistant tuberculosis: first global report. Eur Respir J. 2019;54:1901522. https:/ / doi.org/ 10.1183/13993003.01522-2019

8. Akkerman O, Aleksa A, Alffenaar JW, Al-Marzouqi NH, Arias-Guillén M, Belilovski E, et al.; members of the International Study Group on new anti-tuberculosis drugs and adverse events monitoring. Surveillance of adverse events in the treatment of drug-resistant tuberculosis: A global feasibility study. Int J Infect Dis. 2019;83:72-6. https://doi.org/10.1016/j.ijid.2019.03.036

9. Migliori GB, Sotgiu G, Rosales-Klintz S, Centis R, D'Ambrosio L, Abubakar I, et al. ERS/ECDC Statement: European Union standards for tuberculosis care, 2017 update. Eur Respir J. 2018;51:1702678. https://doi.org/ 10.1183/13993003.02678-2017

10. Getahun H, Matteelli A, Abubakar I, Aziz MA, Baddeley A, Barreira D, et al. Management of latent Mycobacterium tuberculosis infection: WHO guidelines for low tuberculosis burden countries. Eur Respir J. 2015;46:1563-76. https://doi.org/10.1183/13993003.01245-2015

11. Lange SJ, Ritchey MD, Goodman AB, Dias T, Twentyman E, Fuld J, et al. Potential indirect effects of the COVID-19 pandemic on use of emergency departments for acute lifethreatening conditions - United States, January-May 2020. MMWR Morb Mortal Wkly Rep. 2020;69:795-800. https://doi.org/10.15585/mmwr.mm6925e2

12. Min Ong CW, Migliori GB, Raviglione $M$, MacGregorSkinner G, Sotgiu G, Alffenaar JW, et al. Epidemic and pandemic viral infections: impact on tuberculosis and the lung. A consensus by the World Association for Infectious Diseases and Immunological Disorders (WAidid), Global Tuberculosis Network (GTN) and members of ESCMID Study Group for Mycobacterial Infections (ESGMYC). Eur Respir J. 2020;2001727. https://doi.org/10.1183/13993003.01727-2020

13. Esmail H, Cobelens F, Goletti D. Transcriptional biomarkers for predicting development of tuberculosis: progress and clinical considerations. Eur Respir J. 2020;55:1901957. https://doi.org/10.1183/13993003.01957-2019

14. Goletti D, Petrone L, Ippolito G, Niccoli L, Nannini C, Cantini F. Preventive therapy for tuberculosis in rheumatological patients undergoing therapy with biological drugs. Expert Rev Anti Infect Ther. 2018;16:501-12.

https:/ / doi.org/10.1080/14787210.2018.1483238

15. TB Surveillance in the COVID-19 epidemic: national monthly report (provisional data): 1 January 2019 to 31 May 2020 [cited 2020 Jul 23]. https:/ / www.gov.uk/government/statistics/tuberculosis-in-england-quarterly-reports

Address for correspondence: Catherine Ong, National University of Singapore, Division of Infectious Diseases, Department of Medicine, Yong Loo Lin School of Medicine, 1E Kent Ridge Rd, 10th Fl Tower Block, Singapore 119228; email: catherine_wm_ong@nuhs.edu.sg

\section{EID SPOTLIGHT TOPIC: Tuberculosis}

World TB Day, falling on March 24th each year, is designed to build public awareness that tuberculosis today remains an epidemic in much of the world, causing the deaths of nearly one-and-a-half million people each year, mostly in developing countries. It commemorates the day in 1882 when Dr Robert Koch astounded the scientific community by announcing that he had discovered the cause of tuberculosis, the TB bacillus. At the time of Koch's announcement in Berlin, TB was raging through Europe and the Americas, causing the death of one out of every seven people. Koch's discovery opened the way towards diagnosing and curing TB.

Click on the link below to access Emerging Infectious Diseases articles and podcasts, and to learn more about the latest information and emerging trends in TB.

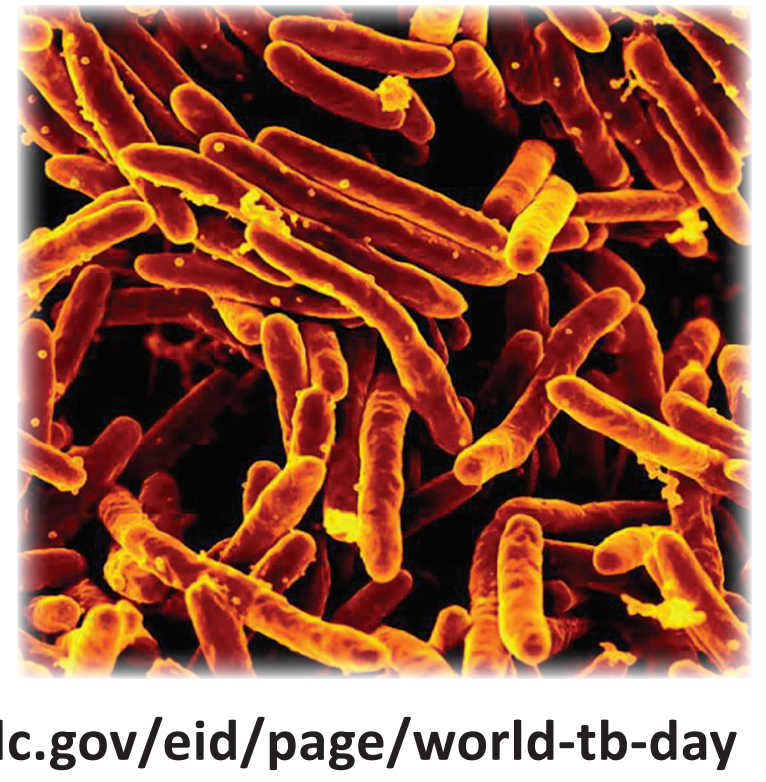

